\title{
Ringraziamenti della redazione ai Referee per l'anno 2012
}

Per l'aiuto fornito alla redazione nel processo di selezione e referaggio degli articoli pubblicati e per il dialogo costruttivo intrattenuto con gli autori durante l'anno 2012, si ringraziano:

$\begin{array}{ll}\text { Bruno Anastasia } & \text { Enzo Rullani } \\ \text { Giancarlo Corò } & \text { Pierangelo Spano } \\ \text { Roberto Grandinetti } & \text { Ugo Trivellato } \\ \text { Nicoletta Masiero } & \text { Federica Vanin } \\ \text { Patrizia Messina } & \end{array}$

ECONOMIA E SOCIETÀ REGIONALE-117 (3) 2012 


\section{COMUNICAZIONE AGLI AUTORI di ECONOMIA E SOCIETÀ REGIONALE}

In seguito all'evoluzione dei criteri di valutazione della ricerca scientifica, FrancoAngeli ha aderito, dal 2012, a CrossRef e tutte le pubblicazioni periodiche vengono registrate in CrossRef (tramite mEDRA http://www.medra.org/it/index.htm, Agenzia di registrazione Doi per l'Italia e per i paesi dell'Europa Occidentale). Tale registrazione consente alle pubblicazioni di entrare nel servizio di CrossLinking. Questo servizio crea una rete di citazioni incrociate, navigabili online, tra le pubblicazioni degli editori che vi aderiscono, incrementando la visibilità e l'accesso ai contenuti citati da ciascun editore. Il CrossLinking mette a disposizione dell'editore e degli Autori il servizio di Cited-by che permette di monitorare l'impatto della pubblicazione sulla comunità editoriale internazionale, calcolando le citazioni effettuate e specificandone la provenienza.

CrossRef chiede, a coloro che aderiscono, di registrare i Doi sulle proprie pubblicazioni rendendole così facilmente citabili dagli altri editori e ricercatori i quali, al contempo, si impegnano a citarsi reciprocamente usando il Doi. Vengono così garantiti link permanenti in modo tale che da ogni riferimento sia possibile risalire alla fonte. Il risultato è una rete di citazioni incrociate, navigabili online, tra le pubblicazioni che incrementa la visibilità e l'accesso ai contenuti citati da ciascun editore.

Nata nel 2002, CrossRef gestisce oggi metadati relativi a quasi 50 milioni di contenuti di cui 25.467 journal, 176.231 libri e 21.550 atti di conferenze, lavorando con un network di 3.594 editori e pubblicazioni che spaziano dal XVII secolo (circa 1.700) fino ai giorni nostri (per il XXI secolo la quota è già quasi 14 milioni

La redazione informa perciò gli Autori che dal n. 1 del 2013 Economia e società regionale adotterà il sistema basato sul Doi (Digital object identifier), uno standard che consente l'identificazione univoca e duratura, all'interno della rete, di una entità oggetto di proprietà intellettuale. A ciascun oggetto, tramite il Doi, vengono associati i relativi dati di riferimento (metadati).

Per poter utilizzare questi servizi è necessario che gli Autori adottino un metodo bibliografico appropriato, riportando i riferimenti bibliografici in un apposito paragrafo alla fine di ogni saggio e completando le singole voci con i codici Doi, quando questi siano attribuiti, riportandoli come nell'esempio dato.

Arts W., Gelissen J. (2002). Three worlds of welfare capitalism or more? A state of the art report. Journal of European Social Policy, 12: 137-158, doi: $10.1177 / 0952872002012002114$

Per la ricerca dei codici Doi gli autori possono utilizzare l'apposita mascherina: http://www.medra.org/en/search.htm messa a disposizione da Medra per le pubblicazioni con essa registrate.

Registrandosi alla pagina web: http://www.crossref.org/requestaccount/, è possibile ottenere i Doi disponibili, sarà poi possibile utilizzare la piattaforma:

http://www.crossref.org/SimpleTextQuery/. 
Per interrogare il sistema sarà necessario inserire la mail con la quale si è effettuata la registrazione e, quindi, copiare dai file i riferimenti bibliografici saggio per saggio e incollarli all'interno della maschera predisposta. Dopo aver selezionato l'opzione "List all possible DOIs per reference" avviare la ricerca. CrosRef restituirà l'elenco delle pubblicazioni inserite aggiungendo i codici Doi disponibili.

L'editing suggerito è il seguente:

Clow GD, McKay CP, Simmons Jr. GM, and Wharton RA, Jr. 1988. Climatological observations and predicted sublimation rates at Lake Hoare, Antarctica. Journal of Climate 1:715-728.

La piattaforma elabora anche altre tipologie di editing bibliografico annullando però l'uso dei corsivi. Si consiglia quindi di utilizzare la piattaforma prima di aver effettuato l'uniformazione redazionale.

Non appena i riferimenti bibliografici della rivista saranno uniformati in base a questa procedura FrancoAngeli caricherà i file sulla piattaforma di Cross Linkin

La redazione invita gli Autori a uniformare i riferimenti bibliografici agli esempi che seguono

\section{Volume:}

Beck A.T., Rush A.J., Shaw B.F. and Emery G. (1979). Cognitive Therapy of Depression. New York: Guilford Press (trad. it.: Terapia cognitiva della depressione. Torino: Boringhieri, 1987)

\section{Curatela, un autore:}

Merini A., a cura di (1977). Psichiatria nel territorio. Milano: Feltrinelli

\section{Curatela, più autori:}

Boltanski L., Claverie E., Offenstadt N. and Van Damme S., a cura di (2007). Affaires, scandales et grandes causes. De Socrate à Pinochet. Paris: Stock

\section{Saggio da curatela:}

Liotti G. (1985). Un modello cognitivo-comportamentale dell'agorafobia. In: Guidano V.F. e Reda M.A., a cura di, Cognitivismo e psicoterapia. Milano: FrancoAngeli

Beebe B. (1983). Mother-infant mutual influence and precursors of self and object representation. In: Masling J., editor, Empirical Studies of Psychoanalytic Theories. Vol. 2. Hillsdale, NJ: Analytic Press

\section{Saggio da rivista:}

Ferrandes G., Mandich P. (2012). Riflessioni sulla medicina predittiva e sulla necessità di integrazione delle discipline: proposta di un modello di consulenza genetica integrata. Psicologia della salute, 3: 11-28, doi: 10.3280/PDS2012003002

\section{Testo non pubblicato:}

Benedetti G. (1988). "Intervento nel dibattito sulla relazione di John Gunderson al Convegno Internazionale New Trends in Schizophrenia", Bologna, 14-17 aprile (incisione su nastro) 


\section{Volume o articolo da sito Internet:}

Si seguono le stesse indicazioni come nel caso di volumi e articoli stampati, con l'aggiunta di: testo disponibile al sito: http://www... e la data di consultazione

La redazione ricorda che gli articoli, inviati a ires@ veneto.cgil.it, devono essere accompagnati da una lettera di liberatoria in cui l'Autore concede alla Direzione della rivista l'esercizio esclusivo di tutti i diritti di sfruttamento economico sull'articolo, senza limiti di spazio ed entro i limiti temporali massimi riconosciuti dalla normativa vigente (attualmente 20 anni) e con tutte le modalità e le tecnologie attualmente esistenti e/o in futuro sviluppate. Si intende pertanto compresa, inter alia, la riproduzione in ogni modo e forma, comunicazione - ivi inclusi, a titolo esemplificativo ma non esaustivo, anche i diritti di sfruttamento patrimoniale a mezzo di canali digitali interattivi (con qualsiasi modalità attualmente nota od in futuro sviluppata) - e distribuzione di cui l'articolo è suscettibile. Parimenti concede alla Direzione in esclusiva il diritto di tradurre, riprodurre, distribuire, comunicare l'articolo in qualsiasi lingua, in qualsiasi modo e forma, i diritti di sfruttamento pubblicitario, il diritto di cedere a terzi i diritti loro spettanti sull'articolo, in tutto o in parte, sia a titolo oneroso che a titolo gratuito, ecc., senza limiti di spazio ed entro i limiti temporali massimi riconosciuti dalla normativa vigente (attualmente, 20 anni) e con tutte le modalità e tecnologie attualmente esistenti e/o in futuro sviluppate.

Gli articoli proposti alla redazione* (max. 60.000-60.000 battute) devono contenere:

- titolo in lingua italiana e in lingua inglese ( $\max 50-60$ battute)

- abstract in lingua italiana e in lingua inglese di circa 1000 battute

- parole chiave e key words

- codici Jel (http://www.aeaweb.org/journal/jel_class_system.html\#H $>* *$.

Le nuove indicazioni sono reperibili sul sito alla pagina http://www.francoangeli.it/riviste/sommario.asp?ID Rivista=14\&lingua=it

* Gli articoli pervenuti alla rivista sono sottoposti a referaggio anonimo. I Referee valutano i lavori proposti e redigono la scheda predisposta dalla redazione. Qualora il loro giudizio complessivo non sia positivo, l'articolo verrà respinto. In caso contrario e se sono richieste modifiche, la scheda di valutazione viene inviata all'autore in forma anonima. Sulla base delle osservazioni ricevute, l'autore, se ritiene, può rivedere il testo e inoltrare alla rivista la versione emendata, che viene trasmessa agli stessi Referee per l'approvazione finale, senza la quale l'articolo non verrà pubblicato.

** Economia e società regionale-Oltre il ponte (II serie) è stata inserita dalla American Economic Association (AEA) fra le riviste indicizzate in EconLit/Journal of Economic Literature (JEL), la banca dati prodotta dall'AEA che comprende oltre mezzo milione di record bibliografici relativi a monografie, articoli di periodici e di opere collettive, dissertazioni, recensioni e working papers nell'ambito della letteratura economica internazionale, con una copertura cronologica che si estende dal 1969 all'anno corrente, aggiornata mensilmente In conformità alle norme per certificazione rispettate dalle riviste indicizzate da Econlit, gli articoli pubblicati nella sezione Saggi e ricerche devono portare l'indicazione dei codici JEL reperibili su 〈http://www.aeaweb.org/journal/jel_class_system.php〉. 